\title{
A general structure theorem for the nash equilibrium correspondence
}

Citation for published version (APA):

Predtetchinski, A. (2006). A general structure theorem for the nash equilibrium correspondence.

METEOR, Maastricht University School of Business and Economics. METEOR Research Memorandum No. 010 https://doi.org/10.26481/umamet.2006010

Document status and date:

Published: 01/01/2006

DOI:

10.26481/umamet.2006010

Document Version:

Publisher's PDF, also known as Version of record

\section{Please check the document version of this publication:}

- A submitted manuscript is the version of the article upon submission and before peer-review. There can be important differences between the submitted version and the official published version of record.

People interested in the research are advised to contact the author for the final version of the publication, or visit the DOI to the publisher's website.

- The final author version and the galley proof are versions of the publication after peer review.

- The final published version features the final layout of the paper including the volume, issue and page numbers.

Link to publication

\footnotetext{
General rights rights.

- You may freely distribute the URL identifying the publication in the public portal. please follow below link for the End User Agreement:

www.umlib.nl/taverne-license

Take down policy

If you believe that this document breaches copyright please contact us at:

repository@maastrichtuniversity.nl

providing details and we will investigate your claim.
}

Copyright and moral rights for the publications made accessible in the public portal are retained by the authors and/or other copyright owners and it is a condition of accessing publications that users recognise and abide by the legal requirements associated with these

- Users may download and print one copy of any publication from the public portal for the purpose of private study or research.

- You may not further distribute the material or use it for any profit-making activity or commercial gain

If the publication is distributed under the terms of Article $25 \mathrm{fa}$ of the Dutch Copyright Act, indicated by the "Taverne" license above, 


\title{
A General Structure Theorem for the Nash Equilibrium Correspondence
}

\author{
Arkadi Predtetchinski*†
}

February 24, 2006

\begin{abstract}
We consider $n$-person normal form games where the strategy set of each player is a non-empty compact convex subset of a Euclidean space, and the payoff function of player $i$ is continuous in joint strategies and continuously differentiable and concave in player $i$ 's strategy. No further restrictions (such as multilinearity of the payoff functions or the requirement that the strategy sets be polyhedral) are imposed. We demonstrate that the graph of the Nash equilibrium correspondence on this domain is homeomorphic to the space of games. This result generalizes a well-known structure theorem in Kohlberg and Mertens [7]. It is supplemented by an extension analogous to the unknottedness theorems in Demichelis and Germano ([3] and [4]): the graph of the Nash equilibrium correspondence is ambient isotopic to a trivial copy of the space of games.
\end{abstract}

JEL classification codes: C72.

Keywords: Nash equilibrium correspondence.

${ }^{*}$ Department of Economics, Maastricht University, P.O. Box 616, 6200 MD Maastricht, The Netherlands. E-mail: a.predtetchinski@algec.unimaas.nl

${ }^{\dagger}$ Helpful comments of Jean-Jacques Herings and Hans Peters are gratefully acknowledged. 


\section{Introduction}

This paper contributes to the study of the geometry of Nash equilibria. The existing studies of the geometry of Nash equilibria usually consider mixed strategy Nash equilibria on the domain of finite games, i.e. games with finitely many pure strategies. This framework, however, is inadequate for modelling a large number of interesting strategic interactions. Market games, Cournot oligopoly games, location games are examples of games with a continuum of pure strategies and non-linear payoffs. In such games, it is the set of equilibria in pure rather than mixed strategies that is of particular importance to the respective applications. The purpose of this paper to extend the study of the geometry of Nash equilibria on a sufficiently rich domain of games that includes these and similar types of games.

The geometry of Nash equilibria is best understood through the properties of the graph of the equilibrium correspondence. A number of topological characterizations of the graphs of various equilibrium correspondences are well-known in the literature. Thus Kohlberg and Mertens [7] show that the graph of the Nash equilibrium correspondence on the domain of finite games is homeomorphic to a Euclidean space. This result is a game-theoretic analogue of the structure theorem in Balasko [1] who shows that the graph of the Walrasian equilibrium correspondence is homeomorphic to a Euclidean space. A topological characterization of the pseudo-equilibrium manifold in economies with incomplete markets is given in Zhou [9]. In Demichelis, Ritzberger, and Swinkels [5] the graph of subgame perfect equilibrium correspondence is shown to be homeomorphic with the underlying space of perfect information games.

This paper provides a topological characterization of the Nash equilibrium correspondence in a very general setup. We consider the space of normal form games as parameterized by the payoff functions. It is assumed that the strategy set of each player is a non-empty compact convex subset of a Euclidean space, and the payoff function of player $i$ is continuous in joint strategies and concave and continuously differentiable in the own strategies of player $i$. No further restrictions (such as multilinearity of the payoff functions or the requirement that the strategy sets be polyhedral) are imposed. It is demonstrated that the graph of the Nash equilibrium correspondence on this domain is homeomorphic to the underlying space of games. Furthermore, the constructed homeomorphism preserves the subspace of expected utility functions, implying (the first part of) the structure theorem in [7] as a corollary.

Multiplicity of equilibria is the main reason why the topological characterizations such as the one in the present paper are not easy to obtain. Even in one-player games as considered in Section 2, where a Nash equilibrium is simply a maximum of a payoff function, the characterization is non-trivial due to the possibility of multiple maxima. In the general case of more than one player the second source of multiplicity of equilibria appears: the coordination problem may result in multiple equilibria even if the best responses are always unique.

It turns out that the geometry of Nash equilibrium in decision problems (i.e. one-player games) is similar to the geometry of subgame perfect equilibrium on the domain of perfect 
information games (see [5]). Indeed, the only source of multiplicity of equilibria in such games is the indeterminateness of the best response due to indifferences in the preferences over terminal outcomes. In both cases the equilibrium correspondence is almost everywhere a continuous single-valued function that occasionally makes a vertical step and even a small perturbation of the ambient space is sufficient to make the graphs of these correspondences look like the graphs of single-valued functions. These results cannot be extended to games with many players. In the general case, only a sufficiently large perturbation of the ambient space can deform the graph of the Nash equilibrium correspondence to a graph of a singlevalued map.

The importance of the structure theorems like the one developed in this paper lies in their extensions and the application to dynamics of equilibria. In Section 4 we develop an extension of the structure theorem in the spirit of the so-called unknottedness theorem in Demichelis and Germano [3, 4]. We show that not only does the graph of the Nash equilibrium correspondence have an intrinsic structure of the space of games, but it can be continuously deformed within its ambient space (games times strategies) to a graph of a single-valued function. It follows as a corollary that there exists a proper homotopy of the the projection map to the identity map, a result analogous to the second part of the structure theorem in [7]. For finite games the unknottedness result is known to have a number of important implications for the dynamics whose rest points are equilibria. For example, it implies that any two Nash dynamics are homotopic within the set of Nash dynamics and that the degree and the index of any two equilibria are equal. Extending the results in $[3,4]$ to larger domains of games such as those studied in this paper is an interesting direction for future research.

Section 5 develops a version of a structure theorem for a larger domain of games than that treated in Sections 3 and 4. We abandon the assumption of differentiability of payoffs and consider the space of games where the payoff function of player $i$ is continuous in joint strategies and concave in the own strategies of player $i$. This domain of games requires different tools than the smaller domain treated in Section 3. In particular, it makes it necessary that the payoff functions be well-defined on a neighborhood of the strategy space. Nevertheless the space of games where differentiability is not imposed is sufficiently interesting to warrant a special consideration. We show that the graph of the Nash equilibrium correspondence on this domain is homeomorphic to the space of games and that it is ambient isotopic to a graph of a single-valued function.

\section{The one-player case}

Let $X$ be a non-empty compact convex subset of some Euclidean space. Let $\mathscr{F}$ be the space of all continuous concave functions on $X$. The set $\mathscr{F}$ is endowed with the compact open topology. It is a topology generated by the subbase consisting of all sets $\{f \in \mathscr{F} \mid$ $f(x) \in E$ for all $x \in C$ \} for $C$ a compact subset of $X$ and $E$ an open subset of $\mathbb{R}$.

In this section we consider one-player games where the player's strategy set is $X$ and his payoff function is an element $f$ of the space $\mathscr{F}$. A Nash equilibrium of the game $f$ 
is a strategy that maximizes the payoff function $f$ on the set $X$. The Nash equilibrium correspondence assigns to each $f \in \mathscr{F}$ the set of Nash equilibria of the game $f$. Thus the graph of the Nash equilibrium correspondence is the subspace

$$
\mathscr{N}=\{(f, x) \in \mathscr{F} \times X \mid x \text { maximizes } f \text { on } X\} .
$$

of $\mathscr{F} \times X$

Figure 1 illustrates the Nash equilibrium correspondence on the domain of one-player games. For a given function $f \in \mathscr{F}$ the set of maximizers of $f$ is a convex set, and it is a singleton if $f$ is strictly convex. Thus the Nash equilibrium correspondence is almost always a continuous single-valued function that occasionally makes a vertical step. Vertical step occurs whenever a payoff function has a flat section that produces multiple maxima. It is easy to see that even a small deformation of the graph of the Nash equilibrium correspondence makes it look like a graph of a single-valued continuous function $\mathscr{F} \longrightarrow X$. A dashed line in Figure 1 illustrates a possible deformation. The deformations of the graph of the Nash equilibrium correspondence are treated more formally in Section 4.

The possibility of multiple maxima makes the problem of finding a homeomorphism of $\mathscr{N}$ with $\mathscr{F}$ non-trivial. For instance, there does not exist a homeomorphism that agrees with the natural projection on the subspace of strictly concave functions. To illustrate this point, consider a subspace $\overline{\mathscr{F}}$ of $\mathscr{F}$ consisting of all strictly concave functions, and a subspace $\mathscr{N} \cap(\overline{\mathscr{F}} \times X)$ of $\mathscr{N}$ denoted by $\overline{\mathscr{N}}$. Since each function in $\overline{\mathscr{F}}$ has a unique maximum, the natural projection $\bar{\pi}: \overline{\mathscr{N}} \longrightarrow \overline{\mathscr{F}}$ that sends a pair $(f, x)$ to the function $f$ is a homeomorphism. It cannot be extended to a homeomorphism between $\mathscr{N}$ and $\mathscr{F}$, however: as $\overline{\mathscr{N}}$ is a dense subset of $\mathscr{N}$, the only continuous extension of $\bar{\pi}$ to the space $\mathscr{N}$ is the natural projection $\pi: \mathscr{N} \longrightarrow \mathscr{F}$ that fails to be injective outside $\overline{\mathscr{F}}$.

Given $x \in X$, let $l_{x} \in \mathscr{F}$ denote the linear function defined by the equation $l_{x}(z)=$ $\langle x, z\rangle$ and let $q \in \mathscr{F}$ be a strictly concave function given by $q(z)=-\frac{1}{2}\langle z, z\rangle$. Consider the map

$$
\begin{gathered}
\mathscr{N} \stackrel{\eta}{\longrightarrow} \mathscr{F} \\
(f, x) \mapsto f+l_{x} .
\end{gathered}
$$

Theorem 1 The map $\eta$ is a homeomorphism. Furthermore, suppose that $\overline{\mathscr{F}}$ is one of the following subspaces of $\mathscr{F}$ : the subspace of linear functions, the subspace of strictly concave functions, or the subspace of $C^{r}$-functions. Let $\overline{\mathscr{N}}$ denote the subspace $\mathscr{N} \cap(\overline{\mathscr{F}} \times X)$ of $\mathscr{N}$. Then $\eta$ is a homeomorphism of pairs $(\mathscr{N}, \overline{\mathscr{N}}) \longrightarrow(\mathscr{F}, \overline{\mathscr{F}})$.

Proof. Let the mapping $\varphi$ be defined as follows:

$$
\begin{array}{r}
\mathscr{F} \stackrel{\varphi}{\longrightarrow} \mathscr{F} \times X \\
f \mapsto\left(f-l_{x}, x\right),
\end{array}
$$

where $x$ is the unique maximizer of the strictly concave function $(f+q)$ on the set $X$. First we show that $\varphi$ maps into the set $\mathscr{N}$. 
Thus let $x$ be the maximizer of $(f+q)$ on $X$. We must show that $x$ is a maximizer of the function $\left(f-l_{x}\right)$. Recall that a concave function $u: X \longrightarrow \mathbb{R}$ attains its maximum at point $x$ if and only if its directional derivative $u^{\prime}(x ; d)$ at point $x$ in any direction $d \in X-\{x\}$ is non-positive. A direct computation shows that $(f+q)^{\prime}(x ; d)=f^{\prime}(x ; d)-l_{x}(d)=$ $\left(f-l_{x}\right)^{\prime}(x ; d)$. This implies that the function $\left(f-l_{x}\right)$ attains its maximum on the set $X$ at the point $x$, as desired.

Clearly, $\eta \circ \varphi$ is the identity map on the set $\mathscr{F}$. To see that $\varphi \circ \eta$ is the identity on $\mathscr{N}$, let $(f, x)$ be a point in $\mathscr{N}$. Then $(\varphi \circ \eta)(f, x)=\left(f+l_{x}-l_{\bar{x}}, \bar{x}\right)$, with $\bar{x}$ being a point of $X$ where the function $\left(f+l_{x}+q\right)$ attains its maximum. By direct computation, the function $\left(l_{x}+q\right)$ attains its maximum at the point $x$. Furthermore, the function $f$ attains its maximum at $x$ because $(f, x) \in \mathscr{N}$. It follows that the function $\left(f+l_{x}+q\right)$ attains its maximum at $x$. Because it has only one maximizer, $x=\bar{x}$, and $(\varphi \circ \eta)(f, x)=(f, x)$, as desired.

The map $\eta$ is continuous since it is a composite of continuous maps. In particular, $x \mapsto l_{x}$ is a continuous map of $X$ into $\mathscr{F}$ and the addition of maps is a continuous operation with respect to the chosen topology on $\mathscr{F}$. Likewise, the map $\varphi$ is a composite of continuous maps. In particular, the map that sends each strictly concave function of $\mathscr{F}$ to its unique maximizer on $X$ is a continuous map. Thus we have proved that $\eta$ is a homeomorphism, with $\varphi$ being its inverse.

Finally, if the function $f$ is linear (concave, or $C^{r}$-differentiable), then so is the function $\left(f+l_{x}\right)$ and the function $\left(f-l_{x}\right)$. Thus, both maps $\eta$ and $\varphi$ preserve the linearity, strict concavity and $C^{r}$ - differentiability of the functions. The second part of the theorem follows.

Given $t \in[0,1]$ consider the map $\eta_{t}: \mathscr{N} \longrightarrow \mathscr{F}$ defined by the equation $\eta_{t}(f, x)=$ $f+t l_{x}$. In particular $\eta_{0}=\pi$ and $\eta_{1}=\eta$. An argument similar to the proof of Theorem 1 shows $\eta_{t}$ to be a homeomorphism for each $t \in(0,1]$. Thus the projection map can be arbitrarily closely approximated by homeomorphisms. This property is peculiar to decision problems and cannot be extended to a general case with many players.

\section{The general structure theorem}

In this section we develop a structure theorem for $n$-person games. As in the previous section, the strategy set of each player is fixed, and the games are parameterized by the payoff functions. It is required that a payoff function of player $i$ be continuous in the joint strategies and be concave and continuously differentiable in the own strategy of player $i$. We show there is a homeomorphism of the Nash equilibrium set with the underlying space of games. Furthermore, the constructed homeomorphism preserves the subspace of games where all payoff functions are expected utility functions. As a corollary, we obtain (the first part of) Theorem 1 in [7]: the space of games where the payoff functions are all expected utility functions is homeomorphic to a Euclidean space.

Let the strategy set $X_{i}$ of player $i \in N$ be a non-empty compact convex set of the 
$k_{i}$-dimensional Euclidean space. Let $X$ denote the product space $X_{1} \times \cdots \times X_{n}$. Let $k=k_{1}+\cdots+k_{n}$. Let $\mathscr{F}_{i}$ be the set of payoff functions of player $i$. This is a set of continuous functions $u_{i}: \mathbb{R}^{k} \longrightarrow \mathbb{R}$ such that $u_{i}$ is concave and continuously differentiable in the own strategy $z_{i}$ of player $i$. Let $d u_{i}\left(x_{i}, x_{-i}\right)$ denote the gradient of $u_{i}$ with respect to $z_{i}$ at point $x$. The set $\mathscr{F}_{i}$ is endowed with the $C^{1}$-compact-open topology. Its subbase consists of all sets $\left\{u_{i} \in \mathscr{F}_{i} \mid u_{i}(x) \in E\right.$ and $d u_{i}\left(x_{i}, x_{-i}\right) \in E^{\prime}$ for all $\left.x \in C\right\}$ for $C$ a compact subset of $\mathbb{R}^{k}, E$ an open subset of $\mathbb{R}$ and $E^{\prime}$ an open subset $\mathbb{R}^{k_{i}}$.

We start out with the set of payoff functions defined throughout the space $\mathbb{R}^{k}$ rather than on $X$ only for mathematical rigor. Insisting that payoffs be defined throughout $\mathbb{R}^{k}$ is inconsequential as the homeomorphism $\eta$ of our main theorem preserves the values of the payoff functions on $X$. This property of $\eta$ allows us to carry over our structure theorem to a setting where two payoff functions are identified as soon as they agree throughout the set $X$ (Theorem 3 ).

The graph of the Nash equilibrium correspondence is the subspace of $\mathscr{F} \times X$ given by

$$
\mathscr{N}=\left\{(u, x) \in \mathscr{F} \times X \mid \begin{array}{c}
x \text { is a Nash equilibrium } \\
\text { of the game } u
\end{array}\right\} .
$$

A function $u_{i} \in \mathscr{F}_{i}$ is said to be linear in the strategy of player $m$ if $u_{i}\left(s z_{m}+t \bar{z}_{m}, z_{-m}\right)=$ $s u_{i}\left(z_{m}, z_{-m}\right)+t u_{i}\left(\bar{z}_{m}, z_{-m}\right)$ for any reals $s$ and $t$. It is said to be affine in the strategy of player $m$ if the equation holds whenever $s+t=1$. Of course, a function $u_{i}$ is linear in $z_{m}$ if it is affine in $z_{m}$ and in addition $u_{i}\left(0_{m}, z_{-m}\right)=0$, where $0_{m}$ is a zero in $\mathbb{R}^{k_{m}}$. For example, a payoff function that does not depend on $z_{m}$ is affine in that variable, but it linear in $z_{m}$ only if it is identically equal to zero.

For each $i$ fix a point $x_{i}^{0}$ in $X_{i}$ arbitrarily. Given $u \in \mathscr{F}$ and $x \in X$ define the functions $l_{i}^{x}, h_{i}^{x} u, g_{i} u \in \mathscr{F}_{i}$ by the equations

$$
\begin{gathered}
l_{i}^{x}(z)=\left\langle x_{i}, z_{i}\right\rangle, h_{i}^{x} u(z)=\left\langle d u_{i}\left(x_{i}, x_{-i}\right)-d u_{i}\left(x_{i}, x_{-i}^{0}\right),\left(z_{i}-x_{i}\right)\right\rangle \text { and } \\
g_{i} u\left(z_{i}\right)=u_{i}\left(z_{i}, x_{-i}^{0}\right)-\frac{1}{2}\left\langle z_{i}, z_{i}\right\rangle .
\end{gathered}
$$

All three functions are independent of the strategies of players other than $i$. The function $l_{i}^{x}$ is linear in the strategy of player $i$, the function $h_{i}^{x} u$ is affine and $g_{i} u\left(z_{i}\right)$ is strictly concave in $z_{i}$. Consider the map $\eta: \mathscr{N} \longrightarrow \mathscr{F}$ that sends a point $(u, x)$ of $\mathscr{N}$ to a point $\bar{u}$ of $\mathscr{F}$ where the payoff $\bar{u}_{i}$ is given by the equation

$$
\bar{u}_{i}=u_{i}+h_{i}^{x} u+l_{i}^{x}
$$

In the one-player case we think of the function $h_{i}^{x} u$ as being identically equal to zero, in which case the map $\eta$ is the same as that considered in the previous section.

Theorem 2 The map $\eta$ is a homeomorphism. Furthermore, let $\overline{\mathscr{F}}_{i}$ be the subspace of $C^{r}$-functions or the subspace of functions that are affine in the strategy of player $m$. Let $\overline{\mathscr{F}}=\overline{\mathscr{F}}_{1} \times \cdots \times \overline{\mathscr{F}}_{n}$, and let $\overline{\mathscr{N}}$ denote the subspace $\mathscr{N} \cap(\overline{\mathscr{F}} \times X)$ of $\mathscr{N}$. Then $\eta$ is a homeomorphism of pairs $(\mathscr{N}, \overline{\mathscr{N}}) \longrightarrow(\mathscr{F}, \overline{\mathscr{F}})$. 
Proof. Consider a map $\varphi: \mathscr{F} \longrightarrow \mathscr{F} \times X$ that sends a point $\bar{u}$ into the pair $(u, x)$, where $x_{i}$ is the unique maximizer of the strictly concave function $g_{i} \bar{u}$ over $X_{i}$ and the payoff function $u_{i}$ is given by the equation $u_{i}=\bar{u}_{i}-h_{i}^{x} \bar{u}-l_{i}^{x}$.

First we show that $\varphi$ maps into $\mathscr{N}$. Thus let $\bar{u}$ be an element of $\mathscr{F}$ and let $\varphi(\bar{u})=(u, x)$. We must show that $x_{i}$ maximizes the payoff function $\bar{u}_{i}$ of player $i$ over the his strategy set given $x_{-i}$. Recall that a concave function attains its maximum over $X_{i}$ at point $x_{i}$ if and only if its gradient at this point is contained in the normal cone of $X_{i}$ at $x_{i}$. Because

$$
u_{i}\left(z_{i}, x_{-i}\right)=\bar{u}_{i}\left(z_{i}, x_{-i}\right)-\left\langle d \bar{u}_{i}\left(x_{i}, x_{-i}\right)-d \bar{u}_{i}\left(x_{i}, x_{-i}^{0}\right),\left(z_{i}-x_{i}\right)\right\rangle-\left\langle x_{i}, z_{i}\right\rangle,
$$

we have the equations $d u_{i}\left(x_{i}, x_{-i}\right)=d \bar{u}_{i}\left(x_{i}, x_{-i}^{0}\right)-x_{i}=d g_{i} \bar{u}\left(x_{i}\right)$. Since $x_{i}$ is a maximizer of the function $g_{i} \bar{u}$ over the set $X_{i}$, the gradient $d g_{i} \bar{u}\left(x_{i}\right)$ is in the normal cone of $X_{i}$ at $x_{i}$. Thus $d u_{i}\left(x_{i}, x_{-i}\right)$ is in the normal cone of $X_{i}$, as desired.

To see that $\eta \circ \varphi$ is the identity on $\mathscr{F}$, let $\bar{u} \in \mathscr{F}, \varphi(\bar{u})=(u, x)$, and $\eta(u, x)=\dot{u}$. Then $u_{i}-\bar{u}_{i}=-h_{i}^{x} \bar{u}-l_{i}^{x}$ and $\dot{u}_{i}-u_{i}=h_{i}^{x} u+l_{i}^{x}$. Thus $\dot{u}_{i}-\bar{u}_{i}=h_{i}^{x} u-h_{i}^{x} \bar{u}$. Now

$$
\begin{gathered}
{\left[h_{i}^{x} u-h_{i}^{x} \bar{u}\right](z)=} \\
=\left\langle d u_{i}\left(x_{i}, x_{-i}\right)-d u_{i}\left(x_{i}, x_{-i}^{0}\right),\left(z_{i}-x_{i}\right)\right\rangle-\left\langle d \bar{u}_{i}\left(x_{i}, x_{-i}\right)-d \bar{u}_{i}\left(x_{i}, x_{-i}^{0}\right),\left(z_{i}-x_{i}\right)\right\rangle= \\
=\left\langle d\left(u_{i}-\bar{u}_{i}\right)\left(x_{i}, x_{-i}\right),\left(z_{i}-x_{i}\right)\right\rangle-\left\langle d\left(u_{i}-\bar{u}_{i}\right)\left(x_{i}, x_{-i}^{0}\right),\left(z_{i}-x_{i}\right)\right\rangle .
\end{gathered}
$$

But $\left(u_{i}-\bar{u}_{i}\right)$ is independent of the strategies of the players other than player $i$, so the two terms of the last expression cancel out. Thus $h_{i}^{x} u-h_{i}^{x} \bar{u}=0$ and $\dot{u}_{i}=\bar{u}_{i}$, as desired.

To see that $\varphi \circ \eta$ is the identity on $\mathscr{N}$, let $(u, x)$ be a point in $\mathscr{N}$. Let $\eta(u, x)=\bar{u}$ and $\varphi(\bar{u})=(\dot{u}, \dot{x})$. Then $\dot{x}_{i}$ is a maximizer of the function $g_{i} \bar{u}$ on $X_{i}$. Observe that

$$
\begin{gathered}
g_{i} \bar{u}\left(z_{i}\right)=g_{i}\left[u_{i}+h_{i}^{x} u+l_{i}^{x}\right]\left(z_{i}\right)= \\
=u_{i}\left(z_{i}, x_{-i}^{0}\right)+\left\langle d u_{i}\left(x_{i}, x_{-i}\right)-d u_{i}\left(x_{i}, x_{-i}^{0}\right), z_{i}-x_{i}\right\rangle+\left\langle x_{i}, z_{i}\right\rangle-\frac{1}{2}\left\langle z_{i}, z_{i}\right\rangle,
\end{gathered}
$$

A direct computation then shows that $d g_{i} \bar{u}\left(x_{i}\right)=d u_{i}\left(x_{i}, x_{-i}\right)$. As $(u, x) \in \mathscr{N}$, the function $u_{i}$ is maximized at $x_{i}$ given $x_{-i}$, therefore $d u_{i}\left(x_{i}, x_{-i}\right)$ is in the normal cone of $X_{i}$ at point $x_{i}$. It follows that the function $g_{i} \bar{u}$ is also maximized at $x_{i}$. As it has but one maximizer, $x_{i}=\dot{x}_{i}$. Now, $\bar{u}_{i}-u_{i}=h_{i}^{x} u+l_{i}^{x}$ and $\dot{u}_{i}-\bar{u}_{i}=-h_{i}^{x} \bar{u}-l_{i}^{x}$. As in the previous paragraph, the difference $\left(h_{i}^{x} u-h_{i}^{x} \bar{u}\right)$ is identically zero, because $\left(u_{i}-\bar{u}_{i}\right)$ is independent of the strategies of $i$ 's opponents. This implies that $\dot{u}_{i}=u_{i}$, as desired.

The maps $\eta$ and $\varphi$ are the composites of continuous maps. In particular, $(u, x) \mapsto h_{i}^{x} u$ is a continuous map of $\mathscr{F} \times X$ into $\mathscr{F}$. Thus $\eta$ is a homeomorphism, with $\varphi$ its inverse.

The functions $h_{i}^{x} u$ and $l_{i}^{x}$ are affine in each variable. As the sum of affine functions is an affine function, the functions $\left(u_{i}+h_{i}^{x} u+l_{i}^{x}\right)$ and $\left(u_{i}-h_{i}^{x} u-l_{i}^{x}\right)$ are affine in the strategy of player $m$ whenever $u_{i}$ is and they are $C^{r}$-functions whenever $u_{i}$ is. The second part of the theorem follows.

The space $\mathscr{F}_{i}$ consists of payoff functions defined on all of the Euclidean space $\mathbb{R}^{k}$. Thus the map $\eta$ treats the payoff functions $u$ and $\bar{u}$ as different points of $\mathscr{F}$ even when differ only outside $X$. We now reestablish our main result for a space of games where two payoff 
functions are identified whenever they agree on the set $X$ of joint strategies. To this end we introduce an equivalence relation on $\mathscr{F}$ by letting $u \sim \bar{u}$ whenever each $u_{i}$ agrees with $\bar{u}_{i}$ throughout the set $X$. The quotient space $\mathscr{F} / \sim$ is a set of equivalence classes of $\sim$ endowed with an identification topology. Each equivalence class of $\sim$ can be represented by a collection $u$ of continuous functions on $X$, each $u_{i}$ concave and continuously differentiable in the own strategy of player $i$. Indeed, for $U_{i} \in \mathscr{F}_{i}$ the function $u_{i}=U_{i} \mid X$ possesses all these properties, and, conversely, any function $u_{i}: X \longrightarrow \mathbb{R}$ with these properties can be extended to a function $U_{i} \in \mathscr{F}_{i}$. We define an equivalence relation $\sim$ on $\mathscr{N}$ by letting $(u, x) \sim(\bar{u}, \bar{x})$ if $u \sim \bar{u}$ and $x=\bar{x}$.

Theorem 3 The map $\eta$ induces a homeomorphism $(\mathscr{N} / \sim) \longrightarrow(\mathscr{F} / \sim)$.

Proof. We must show that the map $\eta$ and its inverse $\varphi$ both preserve the equivalence relation $\sim$. That is $\eta(u, x) \sim \eta(\bar{u}, \bar{x})$ whenever $(u, x) \sim(\bar{u}, \bar{x})$ and $\varphi(u) \sim \varphi(\bar{u})$ whenever $u \sim \bar{u}$. Thus for $u$ and $\bar{u}$ in $\mathscr{F}$ suppose that $u_{i}\left|X=\bar{u}_{i}\right| X$. Because $x^{0}$ is a point of $X$, $g u_{i}\left|X=g \bar{u}_{i}\right| X$. Furthermore, the scalar product $\left\langle d u_{i}\left(x_{i}, x_{-i}\right),\left(z_{i}-x_{i}\right)\right\rangle$ is the directional derivative of $u_{i}$ with respect to the own strategy of player $i$ in the direction of $\left(z_{i}-x_{i}\right)$. For $x$ is an element of $X$ and $z_{i}$ an element of $X_{i}$, it is entirely determined by the values of the function $u_{i}$ on the set $X$. Therefore $h_{i}^{x} u=h_{i}^{x} \bar{u}$. The result follows.

Corollary 1 Let $\mathscr{F}_{i}^{*}$ be the subspace of payoff functions that are affine in each player's strategy. Let $\mathscr{F}^{*}=\mathscr{F}_{1}^{*} \times \cdots \times \mathscr{F}_{n}^{*}$, and let $\mathscr{N}^{*}$ denote the subspace $\mathscr{N} \cap\left(\mathscr{F}^{*} \times X\right)$ of $\mathscr{N}$. Then $\eta$ induces a homeomorphism of pairs $\left(\mathscr{N} / \sim, \mathscr{N}^{*} / \sim\right) \longrightarrow\left(\mathscr{F} / \sim, \mathscr{F}^{*} / \sim\right)$.

Corollary 1 follows immediately from Theorems 2 and 3. Its significance lies in the fact that it encompasses the structure theorem of Kohlberg and Mertens [7]. To see this, let $X_{i}$ be a unit simplex, interpreted as a set of probability distributions over some set of $k_{i}$ pure strategies. Then each equivalence class of $\mathscr{F}^{*} / \sim$ can be represented by a collection $u$, where $u_{i}: X \longrightarrow \mathbb{R}$ is an expected utility function. As an expected utility function is entirely determined by its values on the set of pure strategies (i.e. the vertices of the simplex $X_{i}$ ), the space $\mathscr{F}^{*} / \sim$ can be identified with the Euclidean space of dimension $n \times k_{1} \times \cdots \times k_{n}$. Thus Corollary 1 tells us that $\mathscr{N}^{*} / \sim$ is homeomorphic with a finitedimensional Euclidean space, which is exactly (the first part of) Theorem 1 in [7].

\section{An extension}

This section provides two extensions of the basic result. The first extension is in the spirit of the so-called unknottedness theorem in Demichelis and Germano [3, 4]. It says that the set $\mathscr{N}$ can be continuously deformed, within its ambient space, to a trivial copy of the space of games. The second extension is analogous to the second part of the structure theorem of Kohlberg and Mertens [7, Theorem 1]. It asserts that the homeomorphism $\eta: \mathscr{N} \longrightarrow \mathscr{F}$ is homotopic to the projection map via a proper homotopy. 
Let the spaces $X, \mathscr{F}$ and $\mathscr{N}$ and the maps $\eta: \mathscr{N} \longrightarrow \mathscr{F}$ and $\varphi: \mathscr{F} \longrightarrow \mathscr{F} \times X$ be as in Section 3 and let $\pi: \mathscr{N} \longrightarrow \mathscr{F}$ be the projection. Let $x^{0}$ be a point of $X$ and let $\mathscr{F}_{0}=\mathscr{F} \times\left\{x^{0}\right\}$ denote a trivial copy of the space of games. Furthermore, for each $i$ let $X_{i}^{\prime}$ be a compact convex set containing $X_{i}$ in its relative interior. In applications, we think of $X_{i}^{\prime}$ as a slightly enlarged copy of $X_{i}$. Let $X^{\prime}$ denote the product space $X_{1}^{\prime} \times \cdots \times X_{n}^{\prime}$. Furthermore, let $\mathscr{E} \prime$ denote the product space $\mathscr{F} \times X^{\prime}$. With this notation, the main result of this section is as follows.

Theorem 4 There exist continuous maps $\psi, \xi:[0,1] \times \mathscr{E}^{\prime} \longrightarrow \mathscr{E}^{\prime}$ such that (a) for each $t \in[0,1]$ the map $\psi_{t}=\psi(t, \cdot): \mathscr{E}^{\prime} \longrightarrow \mathscr{E}^{\prime}$ is a homeomorphism with $\xi_{t}=\xi(t, \cdot): \mathscr{E}^{\prime} \longrightarrow \mathscr{E}^{\prime}$ its inverse, (b) for each $t \in[0,1]$ the map $\psi_{t}$ is the identity map on $\mathscr{F} \times \partial X^{\prime}$, (c) $\psi_{0}$ is the identity map on $\mathscr{E}^{\prime}$, and (d) $\psi_{1}$ is a homeomorphism of pairs $\left(\mathscr{E}^{\prime}, \mathscr{N}\right) \longrightarrow\left(\mathscr{E}^{\prime}, \mathscr{F}_{0}\right)$.

We prove theorem 4 in two steps. The first step is to show that the graph of the Nash equilibrium correspondence can be continuously deformed, within its ambient space $\mathscr{E} \prime$ into a graph of a single-valued continuous function $\mathscr{F} \longrightarrow X$. What drives this result is an obvious observation that the homeomorphism $\eta$ has an extension to the space $\mathscr{F} \times X$. The first step is carried out in Proposition 1. The second step is to prove that the graph of any continuous function $\mathscr{F} \longrightarrow X$ can be continuously deformed within $\mathscr{E} \prime$ into a graph $\mathscr{F}_{0}$ of a constant function $u \mapsto x^{0}$. This is in fact a property of the spaces $X$ and $X^{\prime}$ and is independent of our construction of the homeomorphism $\eta$. This step is carried out in Proposition 3. Taking the composite of the deformation of Proposition 1 with that of Proposition 3 yields a desired deformation of $\mathscr{N}$ into $\mathscr{F}_{0}$ as in Theorem 4 above. Thus the maps $\psi$ and $\xi$ of Theorem 4 are defined by letting $\psi_{t}=\psi_{t}^{\prime} \circ \psi_{t}^{\prime \prime}$ and $\xi_{t}=\xi_{t}^{\prime} \circ \xi_{t}^{\prime \prime}$ where $\psi^{\prime}$ and $\xi^{\prime}$ are as in Proposition 1 and $\psi^{\prime \prime}$ and $\xi^{\prime \prime}$ are as in Proposition 3.

Proposition 1 There exist continuous maps $\psi^{\prime}, \xi^{\prime}:[0,1] \times \mathscr{E}^{\prime} \longrightarrow \mathscr{E}^{\prime}$ such that (a) for each $t \in[0,1]$ the map $\psi_{t}^{\prime}=\psi^{\prime}(t, \cdot): \mathscr{E}^{\prime} \longrightarrow \mathscr{E}^{\prime}$ is a homeomorphism with $\xi_{t}^{\prime}=\xi^{\prime}(t, \cdot): \mathscr{E}^{\prime} \longrightarrow \mathscr{E}^{\prime}$ its inverse, (b) for each $t \in[0,1]$ the map $\psi_{t}^{\prime}$ is the identity map on $\mathscr{F} \times \partial X^{\prime}$, (c) $\psi_{0}^{\prime}$ is the identity map on $\mathscr{E}^{\prime}$, and $(d) \psi_{1}^{\prime}$ is a homeomorphism of pairs $\left(\mathscr{E}^{\prime}, \mathscr{N}\right) \longrightarrow\left(\mathscr{E}^{\prime}, \mathscr{G}\right)$, where $\mathscr{G}$ is a graph of a continuous function $\mathscr{F} \longrightarrow X$.

Proof. Let $r: X^{\prime} \longrightarrow X$ be a continuous map that is an identity on $X$ (i.e. a retract of $X^{\prime}$ to $\left.X\right)$. Let $\epsilon: X^{\prime} \longrightarrow[0,1]$ be continuous map such that $\epsilon(X)=1$ and $\epsilon\left(\partial X^{\prime}\right)=0$. Define the map $\psi_{t}^{\prime}, \xi_{t}^{\prime}: \mathscr{E}^{\prime} \longrightarrow \mathscr{E}^{\prime}$ by letting

$$
\begin{aligned}
& \psi_{t}^{\prime}\left(u, x^{\prime}\right)=\left(\bar{u}, x^{\prime}\right), \text { where } \bar{u}_{i}=u_{i}+s t h_{i}^{x} u+s t l_{i}^{x} \text { with } x=r\left(x^{\prime}\right) \text { and } s=\epsilon\left(x^{\prime}\right), \\
& \xi_{t}^{\prime}\left(\bar{u}, x^{\prime}\right)=\left(u, x^{\prime}\right), \text { where } u_{i}=\bar{u}_{i}-s t h_{i}^{x} u-s t l_{i}^{x} \text { with } x=r\left(x^{\prime}\right) \text { and } s=\epsilon\left(x^{\prime}\right) .
\end{aligned}
$$

To see that the map $\xi_{t}^{\prime} \circ \psi_{t}^{\prime}$ is the identity let $(u, x) \in \mathscr{E}^{\prime}, \psi_{t}\left(u, x^{\prime}\right)=\left(\bar{u}, x^{\prime}\right)$ and $\xi_{t}\left(\bar{u}, x^{\prime}\right)=$ $\left(\dot{u}, x^{\prime}\right)$. Then $\bar{u}_{i}-u_{i}=s t h_{i}^{x} \bar{u}+s t l_{i}^{x}$ and $\dot{u}_{i}-u_{i}=-s t h_{i}^{x} u-s t l_{i}^{x}$. Thus $\dot{u}_{i}-\bar{u}_{i}=s t h_{i}^{x} \bar{u}-s t h_{i}^{x} u$. As in the proof of Theorem 2 one shows that the function $\left(h_{i}^{x} \bar{u}-h_{i}^{x} u\right)$ is identically zero, because the function $\left(\bar{u}_{i}-u_{i}\right)$ only depends on the strategies of player $i$. It then follows that $\dot{u}_{i}=\bar{u}_{i}$, as desired. The proof that $\psi_{t}^{\prime} \circ \xi_{t}^{\prime}$ is the identity is similar. 
Clearly, the maps $\psi_{t}$ and $\xi_{t}$ carry $\mathscr{F} \times \partial X^{\prime}$ identically to itself. It is also clear that $\psi_{0}^{\prime}$ is the identity map on $\mathscr{E}^{\prime}$. Finally, we must show that $\mathscr{G}=\psi_{1}^{\prime}(\mathscr{N})$ is a graph of a continuous function. It is easy to see that if $(u, x) \in \mathscr{N}$ then $\psi_{1}^{\prime}(u, x)=(\eta(u, x), x)$. In particular, the set $\mathscr{G}$ is entirely contained in the set $\mathscr{F} \times X$. As the map $\varphi$ is the inverse of $\eta$, a point $(\bar{u}, x)$ of $\mathscr{F} \times X$ is an element of the set $\mathscr{G}$ if and only if $\varphi(\bar{u})=(u, x)$ for some function $u \in \mathscr{F}$. This shows that $\mathscr{G}$ is a graph of the composite map $\mathscr{F} \longrightarrow \mathscr{F} \times X \longrightarrow X$ where the first map is $\varphi$ and the second map is the projection.

Thus there is a family of sets $\mathscr{N}_{t}=\psi_{t}^{\prime}(\mathscr{N})$ parameterized by $t \in[0,1]$ such that each $\mathscr{N}_{t}$ is homeomorphic to $\mathscr{N}$ with $\mathscr{N}_{0}=\mathscr{N}$ and $\mathscr{N}_{1}=\mathscr{G}$. In this sense, the set $\mathscr{N}$ can be continuously deformed, within its ambient space $\mathscr{E}^{\prime}$ to a graph of a single-valued function. The deformation is illustrated in Figure 1 for the case of one player and in Figure 2 for the general case.

Figure 1 makes it intuitively clear that even a small perturbation suffices to deform $\mathscr{N}$ into a graph of a single-valued function. One can show in fact that the $\mathscr{N}_{t}$ is a graph of some single-valued continuous function for all $t \in(0,1]$. Indeed, there is a commutative diagram

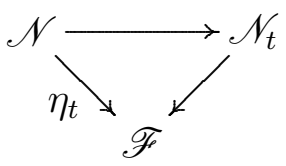

where $\eta_{t}$ is as defined in Section 2, the horizontal map is defined by $\psi_{t}^{\prime}$ and the right-hand vertical map is the projection. As both $\eta_{t}$ for $t \in(0,1]$ and the map $\mathscr{N} \longrightarrow \mathscr{N}_{t}$ defined by $\psi_{t}^{\prime}$ are homeomorphisms, so is the projection map $\mathscr{N}_{t} \longrightarrow \mathscr{F}$. But this means that $\mathscr{N}_{t}$ is a graph of a single-valued continuous function. This result is peculiar to decision problems and cannot be extended to a general case with many players. In the general case, only a sufficiently large perturbation can deform $\mathscr{N}$ into a graph of a single-valued function.

Now we proceed with step two of the proof of our main result: we show that the graph of any continuous function from $\mathscr{F}$ to $X$ is ambient isotopic in $\mathscr{E}^{\prime}$ to a trivial copy of the space of games. This requires the following preliminary result.

Proposition 2 There exist continuous maps $\tau_{i}, \gamma_{i}: X_{i} \times X_{i}^{\prime} \longrightarrow X_{i}^{\prime}$ such that (a) for each $x_{i} \in X_{i}$ the map $\tau_{i}^{x_{i}}=\tau_{i}\left(x_{i}, \cdot\right): X_{i}^{\prime} \longrightarrow X_{i}^{\prime}$ is a homeomorphism with $\gamma_{i}^{x_{i}}=\gamma_{i}\left(x_{i}, \cdot\right)$ : $X_{i}^{\prime} \longrightarrow X_{i}^{\prime}$ its inverse, (b) $\tau_{i}\left(x_{i}, \cdot\right)$ is the identity on the relative boundary of $X_{i}^{\prime}$, (c) $\tau_{i}\left(x_{i}, x_{i}^{0}\right)=x_{i}$, and $(d) \tau_{i}\left(x_{i}^{0}, \cdot\right)$ is the identity map of $X_{i}^{\prime}$.

Proof. First we define the maps $\tau_{i}$ and $\gamma_{i}$ for the special case where $X_{i}^{\prime}$ is a $q$-simplex. Let sd $\partial X_{i}^{\prime}$ be the first barycentric subdivision of the boundary of $X_{i}^{\prime}$. Let $K$ be a subdivision of $X_{i}^{\prime}$ consisting of all simplices of the form $x_{i}^{0} x^{1} \cdots x^{q}$ for $x^{1} \cdots x^{q}$ a simplex of $\operatorname{sd} \partial X_{i}^{\prime}$. Similarly, let $K^{\prime}$ be a subdivision of $X_{i}^{\prime}$ consisting of all simplices $x_{i} x^{1} \cdots x^{q}$ for $x^{1} \cdots x^{q} \in$ $\operatorname{sd} \partial X_{i}^{\prime}$. We define $\tau_{i}^{x_{i}}$ as a simplicial map of $|K|$ to $\left|K^{\prime}\right|$ induced by the vertex map $x_{i}^{0} \mapsto x_{i}$ and $x^{j} \mapsto x^{j}$ for a vertex $x^{j}$ of $\operatorname{sd} \partial X_{i}^{\prime}$. Then $\tau_{i}^{x_{i}}$ is a simplicial homeomorphism. It is illustrated in Figure 3 for the case of $q=1$ and in Figure 4 for the case $q=2$. Its inverse 
is a simplicial map $\gamma_{i}^{x_{i}}:\left|K^{\prime}\right| \mapsto|K|$ induced by a vertex map $x_{i} \mapsto x_{i}^{0}$ and $x^{j} \mapsto x^{j}$ for a vertex $x^{j}$ of $\operatorname{sd} \partial X_{i}^{\prime}$.

In the general case there exists a homeomorphism $h: X_{i}^{\prime} \longrightarrow S$ of $X_{i}^{\prime}$ with a simplex $S$. Of course, this homeomorphism carries the boundary of $X_{i}^{\prime}$ into the boundary of the simplex $S$ and the set $X_{i}$ into the relative interior of $S$. As has been demonstrated in the previous paragraph there exist maps $\tau_{i}, \gamma_{i}: h\left(X_{i}\right) \times S \longrightarrow S$ satisfying the conditions (a)(d) for the pair $\left(h\left(X_{i}\right), S\right)$. It follows that the maps $h^{-1} \tau_{i}(h, h), h^{-1} \gamma_{i}(h, h): X_{i} \times X_{i}^{\prime} \longrightarrow X_{i}^{\prime}$ satisfy the conditions (a)-(d) for the pair $\left(X_{i}, X_{i}^{\prime}\right)$.

Proposition 3 Let $\mathscr{G}$ be a graph of a continuous function $\phi: \mathscr{F} \longrightarrow X$. There exist continuous maps $\psi^{\prime \prime}, \xi^{\prime \prime}:[0,1] \times \mathscr{E}^{\prime} \longrightarrow \mathscr{E}^{\prime}$ such that (a) for each $t \in[0,1]$ the map $\psi_{t}^{\prime \prime}=\psi^{\prime \prime}(t, \cdot): \mathscr{E}^{\prime} \longrightarrow \mathscr{E}^{\prime}$ is a homeomorphism with $\xi_{t}^{\prime \prime}=\xi^{\prime \prime}(t, \cdot): \mathscr{E}^{\prime} \longrightarrow \mathscr{E}^{\prime}$ its inverse, (b) for each $t \in[0,1]$ the map $\psi_{t}^{\prime \prime}$ is the identity on $\mathscr{F} \times \partial X^{\prime}$, (c) $\psi_{0}^{\prime \prime}$ is the identity map on $\mathscr{E}^{\prime}$, and $(d) \psi_{1}^{\prime \prime}$ is a homeomorphism of pairs $\left(\mathscr{E}^{\prime}, \mathscr{G}\right) \longrightarrow\left(\mathscr{E}^{\prime}, \mathscr{F}_{0}\right)$.

Proof. Let the maps $\tau_{i}$ and $\gamma_{i}$ be as in Proposition 2. Let $\phi_{i}$ denote the composite of $\phi$ with the projection $X \longrightarrow X_{i}$. Define now the maps $\psi_{t}^{\prime \prime}, \xi_{t}^{\prime \prime}: \mathscr{E}^{\prime} \longrightarrow \mathscr{E}^{\prime}$ by letting

$$
\begin{aligned}
\psi_{t}^{\prime \prime}\left(u, x^{\prime}\right) & =\left(u, \bar{x}^{\prime}\right), \text { where } \bar{x}_{i}^{\prime}=\gamma_{i}\left((1-t) x_{i}^{0}+t \phi_{i}(u), x_{i}^{\prime}\right), \\
\xi_{t}^{\prime \prime}\left(u, \bar{x}^{\prime}\right) & =\left(u, x^{\prime}\right), \text { where } x_{i}^{\prime}=\tau_{i}\left((1-t) x_{i}^{0}+t \phi_{i}(u), \bar{x}_{i}^{\prime}\right) .
\end{aligned}
$$

The maps $\psi_{t}^{\prime \prime}$ and $\xi_{t}^{\prime \prime}$ are the inverses of each other, because the maps $\gamma_{i}\left(x_{i}, \cdot\right)$ is the inverse of $\tau_{i}\left(x_{i}, \cdot\right)$ for any $x_{i} \in X_{i}$. They carry the set $\mathscr{F} \times \partial X^{\prime}$ identically into itself because $\gamma_{i}\left(x_{i}, \cdot\right)$ and $\tau_{i}\left(x_{i}, \cdot\right)$ are the identity maps on $\partial X_{i}^{\prime}$ for any $x_{i} \in X_{i}$. The maps $\psi_{0}^{\prime \prime}$ and $\xi_{0}^{\prime \prime}$ are the identity maps, because $\gamma_{i}\left(x_{i}^{0}, \cdot\right)$ and $\tau_{i}\left(x_{i}^{0}, \cdot\right)$ are the identity maps of $X_{i}^{\prime}$.

Finally we must show that $\psi_{1}^{\prime \prime}$ induces a homeomorphism of $\mathscr{G}$ with $\mathscr{F}_{0}$. If $(u, x) \in \mathscr{G}$, then $x=\phi(u) \in X$. Therefore, $\psi_{1}^{\prime \prime}(u, x)=\left(u, \bar{x}^{\prime}\right)$, where $\bar{x}_{i}^{\prime}=\gamma_{i}\left(\phi_{i}(u), x_{i}\right)=\gamma_{i}\left(x_{i}, x_{i}\right)=$ $x_{i}^{0}$. Thus $\psi_{1}^{\prime \prime}$ carries $\mathscr{G}$ into $\mathscr{F}_{0}$. Conversely, if $u \in \mathscr{F}$, then $\xi_{1}^{\prime \prime}\left(u, x_{i}^{0}\right)=\left(u, x^{\prime}\right)$, where $x_{i}^{\prime}=\tau_{i}\left(\phi_{i}(u), x_{i}^{0}\right)=\phi_{i}(u)$. Thus $x^{\prime}=\phi(u)$ and $\xi_{1}^{\prime \prime}$ carries $\mathscr{F}_{0}$ into $\mathscr{G}$.

Corollary 2 There exists a proper homotopy map of $\pi$ to $\eta$.

Proof. First we argue that the map $\psi$ of Theorem 4 is a proper map. Consider the maps $\Psi$ and $\Xi$ of $[0,1] \times \mathscr{F} \times X^{\prime}$ to itself defined by the equations $\Psi(t, u, x)=\left(t, \psi_{t}(u, x)\right)$ and $\Xi(t, u, x)=\left(t, \xi_{t}(u, x)\right)$. These maps are continuous and they are the inverses to each other. Now for a subset $K$ of $\mathscr{F} \times X^{\prime}$ we have $\psi^{-1}(K)=\Psi^{-1}([0,1] \times K)=\Xi([0,1] \times K)$. If $K$ is compact, so is the set $[0,1] \times K$. Thus $\psi^{-1}(K)$ is compact, as desired.

Define $\lambda$ as a composite

$$
[0,1] \times \mathscr{N} \longrightarrow[0,1] \times \mathscr{F} \times X^{\prime} \stackrel{\psi}{\longrightarrow} \mathscr{F} \times X^{\prime} \longrightarrow \mathscr{F}
$$

where the first map is the inclusion and the third map is the projection. Now the inclusion map is obviously proper, while the projection $\mathscr{F} \times X^{\prime} \longrightarrow \mathscr{F}$ is a proper map because $X^{\prime}$ 
is a compact set. Thus $\lambda$ is a proper map. Furthermore, $\lambda_{0}=\pi$, because $\psi_{0}$ is the identity map. Furthermore, $\lambda_{1}=\eta$ because $\psi_{1} \mid \mathscr{N}=\eta$. Thus $\lambda$ is a proper homotopy connecting the map $\pi$ to $\eta$.

In the one-player case the homotopy $\lambda$ is such that $\lambda_{t}=\eta_{t}$ for $\eta_{t}$ as in Section 2 and is a homeomorphism for each $t \in(0,1]$.

Theorem 4 is parallel to the results in Demichelis and Germano [3, 4] where an unknottedness theorem is established for Nash equilibria on the domain of normal-form games and for Walrasian equilibria on the domain of exchange economies, respectively. In those settings the unknottedness theorem is known to have a number of important implications for dynamics whose rest points are equilibria. In particular, it implies that the set of Nash dynamics is arcwise connected, i.e. any two dynamics are homotopic within the set of Nash dynamics. This fact in turn implies that an index of any equilibrium is the same for all dynamics. Theorem 4 thus opens up an interesting direction for future research: obtaining the analogous of the results for Nash dynamics on larger domains as consider in this paper.

\section{A different version of the structure theorem}

In this section we develop a version of the structure theorem for a wider domain of games than that considered in Section 3. Specifically, we drop the assumption of differentiability of the payoff functions and consider a space of games where the payoff function of player $i$ is continuous in joint strategies and concave in the own strategy of player $i$. We show that the graph of the Nash equilibrium correspondence on this domain is homeomorphic to the underlying space of games. It is no longer the case, however, that the homeomorphism of the structure theorem preserves an equivalence relation as defined in the preceding section: even when two payoff functions are the same on $X$, their images under the homeomorphism $\eta$ maybe different within $X$.

Let $X_{i}^{\prime}$ be a compact convex set containing $X_{i}$ in its relative interior. The set $\mathscr{F}_{i}$ of payoff functions of player $i$ is a set of continuous functions $u_{i}: X_{i}^{\prime} \longrightarrow \mathbb{R}$ such that $u_{i}$ is concave in the own strategy $z_{i}$ of player $i$. The set $\mathscr{F}_{i}$ is endowed with the compact-open topology, the topology generated by the subbase consisting of all sets $\left\{u_{i} \in \mathscr{F}_{i} \mid u_{i}(x) \in\right.$ $E$ for all $x \in C\}$ for $C$ a compact subset of $X_{i}^{\prime}$ and $E$ an open subset of $\mathbb{R}$. The graph of the Nash equilibrium correspondence is defined as before.

Let $\tau_{i}$ and $\gamma_{i}$ be as in Proposition 2. Given an $x \in X$ let $s_{i}^{x}: X^{\prime} \longrightarrow X^{\prime}$ be a product of the identity map on $X_{i}^{\prime}$ and of the maps $\tau_{m}^{x_{m}}$ for $m \neq i$. Then $s_{i}^{x}$ is a homeomorphism and its inverse is a product of the identity map on $X_{i}^{\prime}$ and the maps $\gamma_{m}^{x_{m}}$ for $m \neq i$. The map $s_{i}^{x^{0}}$ is the identity map and for each $x \in X$ the map $s_{i}^{x_{i}}$ carries a point $\left(z_{i}, x_{-i}^{0}\right)$ into $\left(z_{i}, x_{-i}\right)$.

Let the function $l_{i}^{x} \in \mathscr{F}_{i}$ and the function $g_{i} u \in \mathscr{F}_{i}$ be as in Section 3. Consider the map $\eta: \mathscr{N} \longrightarrow \mathscr{F}$ that sends a point $(u, x)$ into $\bar{u}$, with the payoff function $\bar{u}_{i}$ defined by the equation

$$
\bar{u}_{i}=u_{i} \circ s_{i}^{x}+l_{i}^{x}
$$


Thus the payoff function $\bar{u}_{i}$ is obtained from $u_{i}$ by using homeomorphic transformations $\tau_{m}^{x_{m}}$ of the strategy of player $m$ for $m \neq i$ and adding a term that depends linearly on $i$ 's own strategy. In the one-player case the map $s_{i}^{x}$ is the identity map of $X_{i}^{\prime}$ and the map $\eta$ is the same as in Section 2.

Theorem 5 The mapping $\eta$ is a homeomorphism. Furthermore, let $\overline{\mathscr{F}}_{i}$ be the subspace of $\mathscr{F}_{i}$ consisting of functions $u_{i}$ that are linear in $z_{i}$. Let $\overline{\mathscr{F}}_{=} \overline{\mathscr{F}}_{1} \times \cdots \times \overline{\mathscr{F}}_{n}$, and let $\overline{\mathscr{N}}$ denote the subspace $\mathscr{N} \cap(\overline{\mathscr{F}} \times X)$ of $\mathscr{N}$. Then the mapping $\eta$ is a homeomorphism of $\operatorname{pairs}(\mathscr{N}, \overline{\mathscr{N}}) \longrightarrow(\mathscr{F}, \overline{\mathscr{F}})$.

Proof. Consider the mapping $\varphi: \mathscr{F} \longrightarrow \mathscr{F} \times X$ that sends a point $\bar{u}$ into the point $(u, x)$, where $x_{i}$ is the unique maximizer of the function $g_{i} \bar{u}$ and the payoff function $\bar{u}^{i}$ is defined by the equation

$$
u_{i}=\bar{u}_{i} \circ\left[s_{i}^{x}\right]^{-1}-l_{i}^{x} .
$$

To see that $\varphi$ maps into $\mathscr{N}$, let $u$ be an element of $\mathscr{F}$ and let $\varphi(u)=(\bar{u}, x)$. Recall that a concave function attains its maximum over $X_{i}$ at point $x_{i}$ if and only if its subdifferential at $x_{i}$ has a non-empty intersection with the normal cone of $X_{i}$ at $x_{i}$. Because $\left[s_{i}^{x}\right]^{-1}\left(z^{i}, x_{-i}\right)=\left(z^{i}, x_{-i}^{0}\right)$ for all $z_{i}$, we have $u_{i}\left(z^{i}, x_{-i}\right)=\bar{u}_{i}\left(z^{i}, x_{-i}^{0}\right)-\left\langle x_{i}, z_{i}\right\rangle$. Thus $\partial u_{i}\left(x_{i}, x_{-i}\right)=\partial \bar{u}_{i}\left(x_{i}, x_{-i}^{0}\right)-x_{i}=\partial g_{i} \bar{u}\left(x_{i}\right)$. Then $\partial u_{i}\left(x_{i}, x_{-i}\right)$ has a point in common with the normal cone of $X_{i}$ at $x_{i}$, because $\partial g_{i} \bar{u}\left(x_{i}\right)$ does.

To see that $\eta \circ \varphi$ is the identity map on $\mathscr{F}$, let $(\eta \circ \varphi)(\bar{u})=u$. Then

$$
u_{i}=\left(\bar{u}_{i} \circ\left[s_{i}^{x}\right]^{-1}-l_{i}^{x}\right) \circ s_{i}^{x}+l_{i}^{x}=\bar{u}_{i}-l_{i}^{x} \circ s_{i}^{x}+l_{i}^{x}=\bar{u}_{i},
$$

where the last equality follows from the fact that $l_{i}^{x}$ only depends on $z_{i}$ and $s_{i}^{x}$ is the identity map on $X_{i}^{\prime}$.

To see that $\varphi \circ \eta$ is the identity on $\mathscr{N}$, let $(u, x) \in \mathscr{N}, \eta(u, x)=\bar{u}$ and $\varphi(\bar{u})=(\dot{u}, \dot{x})$. The point $\operatorname{dot}_{i}$ is a maximizer of the function $g_{i} \bar{u}$ on $X_{i}$. Now

$$
g_{i} \bar{u}\left(z_{i}\right)=\left(u_{i} \circ s_{i}^{x}+l_{i}^{x}\right)\left(z_{i}, x_{-i}^{0}\right)-\frac{1}{2}\left\langle z_{i}, z_{i}\right\rangle=u_{i}\left(z_{i}, x_{-i}\right)+\left\langle x_{i}, z_{i}\right\rangle-\frac{1}{2}\left\langle z_{i}, z_{i}\right\rangle .
$$

By direct computation, the function $\left\langle x_{i}, z_{i}\right\rangle-\frac{1}{2}\left\langle z_{i}, z_{i}\right\rangle$ attains its maximum at $x_{i}$. Furthermore, $u_{i}\left(z_{i}, x_{-i}\right)$ reaches a maximum at $x_{i}$, because $(u, x) \in \mathscr{N}$. Thus $g_{i} \bar{u}$ attains a maximum at $x_{i}$. As it has only one maximizer, $x_{i}=\dot{x}_{i}$. It follows that

$$
\bar{u}_{i}=\left(u_{i} \circ s_{i}^{x}+l_{i}^{x}\right) \circ\left[s_{i}^{x}\right]^{-1}-l_{i}^{x}=u_{i} .
$$

Thus $\dot{u}=u$, as desired.

The maps $\eta$ and $\varphi$ are continuous as they are the composites of continuous maps. In particular, the map $x \mapsto u_{i} \circ s_{i}^{x}$ is a continuous map of $X$ to $\mathscr{F}_{i}$. Finally, the mapping $\eta$ preserves the linearity and strict concavity of the payoff functions of player $i$ in $i$ 's own strategy, and so does $\varphi$. The second part of the theorem follows.

The homeomorphism $\eta$ does not preserve the relation $\sim$ as defined in Section 3. Because $s_{i}^{x}$ does not carry $X_{i}$ into itself, the functions $\eta(u, x)$ and $\eta(\bar{u}, x)$ may be different on $X$, 
even when the payoff functions $u$ and $\bar{u}$ agree throughout $X$. We do not know if the quotient space $\mathscr{N} / \sim$ is homeomorphic to the space $\mathscr{F} / \sim$.

Proposition 4 below states the set $\mathscr{N}$ is ambient isotopic with a graph $\mathscr{G}$ of a continuous function $X \longrightarrow \mathscr{F}$ (In fact, a graph of a composite function $\mathscr{F} \longrightarrow \mathscr{F} \times X \longrightarrow X$, where the first map is $\varphi$ and the second map is the projection). Combining this isotopy with that of Proposition 3 yields an exact analogue of Theorem 4.

Proposition 4 There exist continuous maps $\psi^{\prime}, \xi^{\prime}:[0,1] \times \mathscr{E}^{\prime} \longrightarrow \mathscr{E}^{\prime}$ such that (a) for each $t \in[0,1]$ the map $\psi_{t}^{\prime}=\psi^{\prime}(t, \cdot): \mathscr{E}^{\prime} \longrightarrow \mathscr{E}^{\prime}$ is a homeomorphism with $\xi_{t}^{\prime}=\xi^{\prime}(t, \cdot): \mathscr{E}^{\prime} \longrightarrow \mathscr{E}^{\prime}$ its inverse, (b) for each $t \in[0,1]$ the map $\psi_{t}^{\prime}$ is the identity map on $\mathscr{F} \times \partial X^{\prime}$, (c) $\psi_{0}^{\prime}$ is the identity map on $\mathscr{E}^{\prime}$, and (d) $\psi_{1}^{\prime}$ is a homeomorphism of pairs $\left(\mathscr{E}^{\prime}, \mathscr{N}\right) \longrightarrow\left(\mathscr{E}^{\prime}, \mathscr{G}\right)$, where $\mathscr{G}$ is a graph of a continuous function $\mathscr{F} \longrightarrow X$.

Proof. Let $r: X^{\prime} \longrightarrow X$ be a retract of $X^{\prime}$ to $X$ and $\epsilon: X^{\prime} \longrightarrow[0,1]$ be continuous map such that $\epsilon(X)=1$ and $\epsilon\left(\partial X^{\prime}\right)=0$. Define the map $\psi_{t}^{\prime}, \xi_{t}^{\prime}: \mathscr{E}^{\prime} \longrightarrow \mathscr{E}^{\prime}$ by letting

$\psi_{t}\left(u, x^{\prime}\right)=\left(\bar{u}, x^{\prime}\right)$, where $\bar{u}_{i}=u_{i} \circ s_{i}^{x}+e t l_{i}^{x}$ with $e=\epsilon\left(x^{\prime}\right)$ and $x=(1-e t) x^{0}+\operatorname{etr}\left(x^{\prime}\right)$, $\xi_{t}\left(\bar{u}, x^{\prime}\right)=\left(u, x^{\prime}\right)$, where $u_{i}=\bar{u}_{i} \circ\left[s_{i}^{x}\right]^{-1}-e t l_{i}^{x}$ with $e=\epsilon\left(x^{\prime}\right)$ and $x=(1-e t) x^{0}+e t r\left(x^{\prime}\right)$.

To see that the map $\psi_{t}^{\prime} \circ \xi_{t}^{\prime}$ is the identity let $(u, x) \in \mathscr{E}^{\prime}$ and $\left(\psi_{t}^{\prime} \circ \xi_{t}^{\prime}\right)\left(u, x^{\prime}\right)=\left(\dot{u}, x^{\prime}\right)$. Then

$$
\dot{u}_{i}=\left(u_{i} \circ s_{i}^{x}+e t l_{i}^{x}\right) \circ\left[s_{i}^{x}\right]^{-1}-e t l_{i}^{x}=u_{i}+e t l_{i}^{x} \circ\left[s_{i}^{x}\right]^{-1}-e t l_{i}^{x}=u_{i},
$$

where the last equality holds because the function $l_{i}^{x}$ depends only on the own strategy of player $i$ and the function $s_{i}^{x}$ is the identity on the set $X_{i}^{\prime}$. The proof that $\xi_{t}^{\prime} \circ \psi_{t}^{\prime}$ is the identity is similar. To see that (b) holds take a pair $\left(u, x^{\prime}\right) \in \mathscr{F} \times \partial X^{\prime}$ and let $\psi_{t}\left(u, x^{\prime}\right)=\left(\bar{u}, x^{\prime}\right)$. Then $e=\epsilon\left(x^{\prime}\right)=0$ and $x=x^{0}$, and, because $s_{i}^{x^{0}}$ is the identity map, $\bar{u}=u$. A similar argument shows that (c) holds. For condition (d) observe that if $(u, x) \in \mathscr{N}$ then $\psi_{1}^{\prime}(u, x)=(\eta(u, x), x)$. It follows that $\psi_{1}^{\prime}(\mathscr{N})$ is a graph of the composite map $\mathscr{F} \longrightarrow \mathscr{F} \times X \longrightarrow X$ where the first map is $\varphi$ and the second map is the projection.

\section{References}

[1] Yves Balasko. Economic Equilibrium and Catastrophe Theory: An Introduction. Econometrica, 46: 557-569, 1978.

[2] Lawrence E. Blume and William Zame. The Algebraic Geometry of Perfect and Sequential Equilibrium. Econometrica, 62: 783-794, 1994.

[3] Stefano Demichelis and Fabrizio Germano. Some Consequences of the Unknottedness of the Walras Correspondence. Journal of Mathematical Economics, 34: 537-45, 2000. 
[4] Stefano Demichelis and Fabrizio Germano. On (Un)knots and Dynamics in Games. Games and Economic Behavior, 41: 46-60, 2002.

[5] Stefano Demichelis, Klaus Ritzberger, and Jeroen M. Swinkels. The Simple Geometry of Perfect Information Games. Internationa Journal of Game Theory, 32: 315-338, 2004.

[6] Srihari Govindan and Robert Wilson. Direct Proofs of Generic Finiteness of Nash Equilibrium Outcomes. Econometrica, 69: 765-769, 2001.

[7] Elon Kohlberg and Jean-François Mertens. On the Strategic Stability of Equilibria. Econometrica, 54: 1003-1037, 1986.

[8] R. Tyrrell Rockafellar. Convex Analysis. Princeton University Press, Princeton, New Jersey, 1997.

[9] YuqIng Zhou. Genericity Analysis on the Pseudo-Equilibrium Manifold. Journal of Economic Theory, 73: 79-92, 1997. 


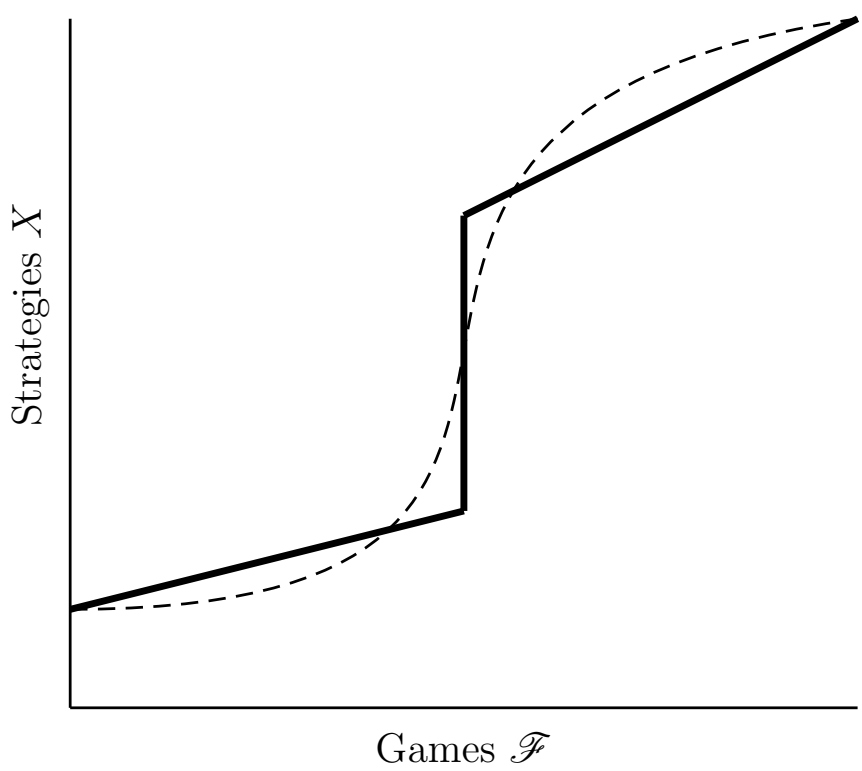

Figure 1: The set $\mathscr{N}$ (bold) and its deformation $\mathscr{G}$ (dashed) in the one-player case. 


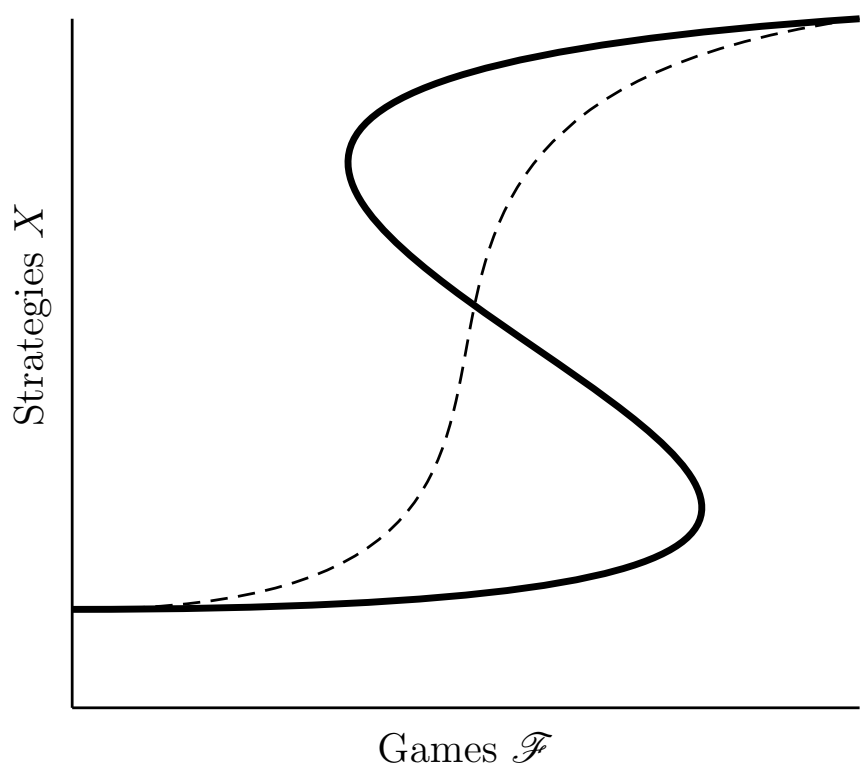

Figure 2: The set $\mathscr{N}$ (bold) and its deformation $\mathscr{G}$ (dashed) in the case of many players. 


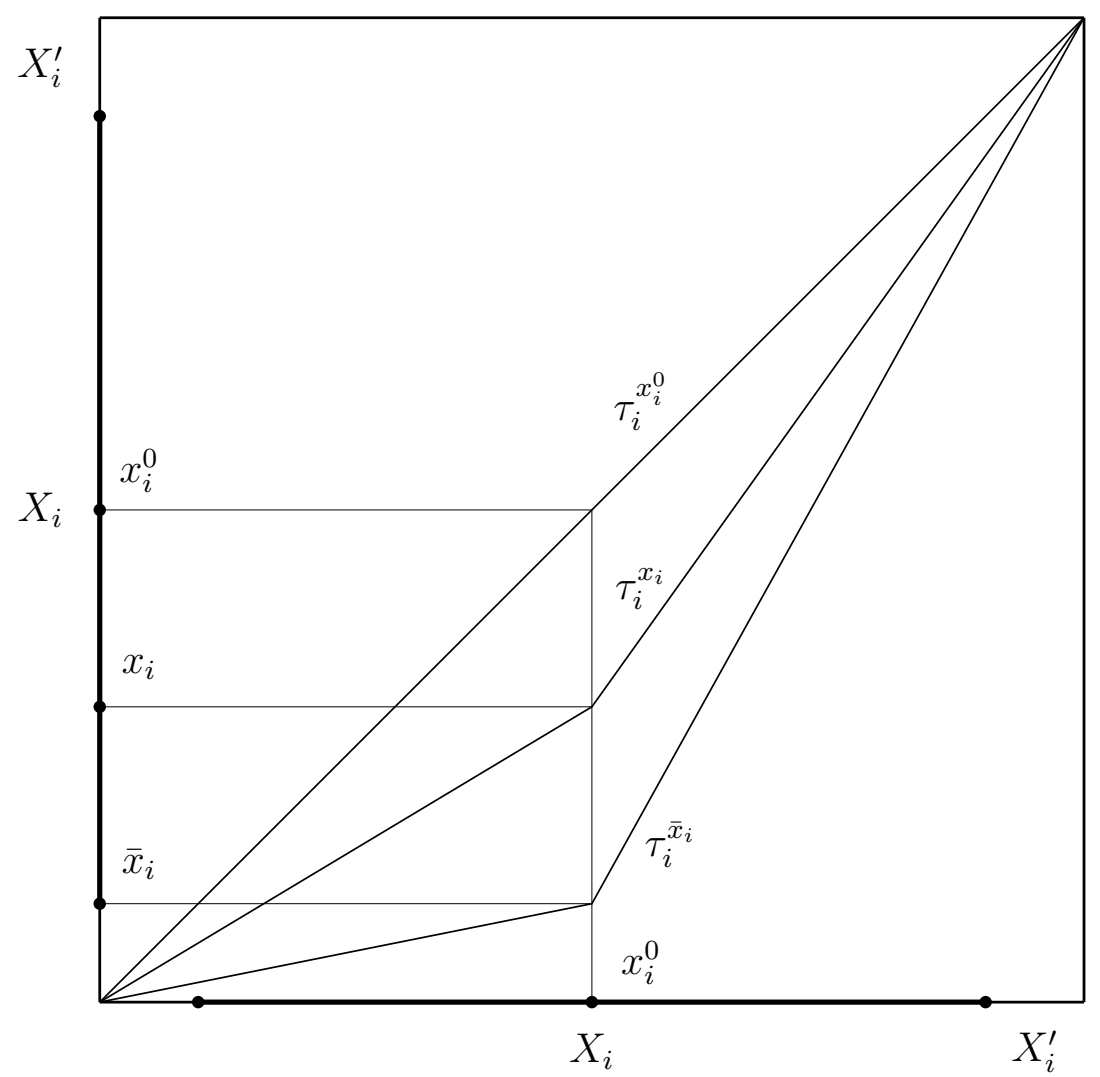

Figure 3: The family of homeomorphisms $\tau_{i}^{x_{i}}=\tau\left(x_{i}, \cdot\right)$ when $X_{i}^{\prime}$ is a 1 -simplex. 


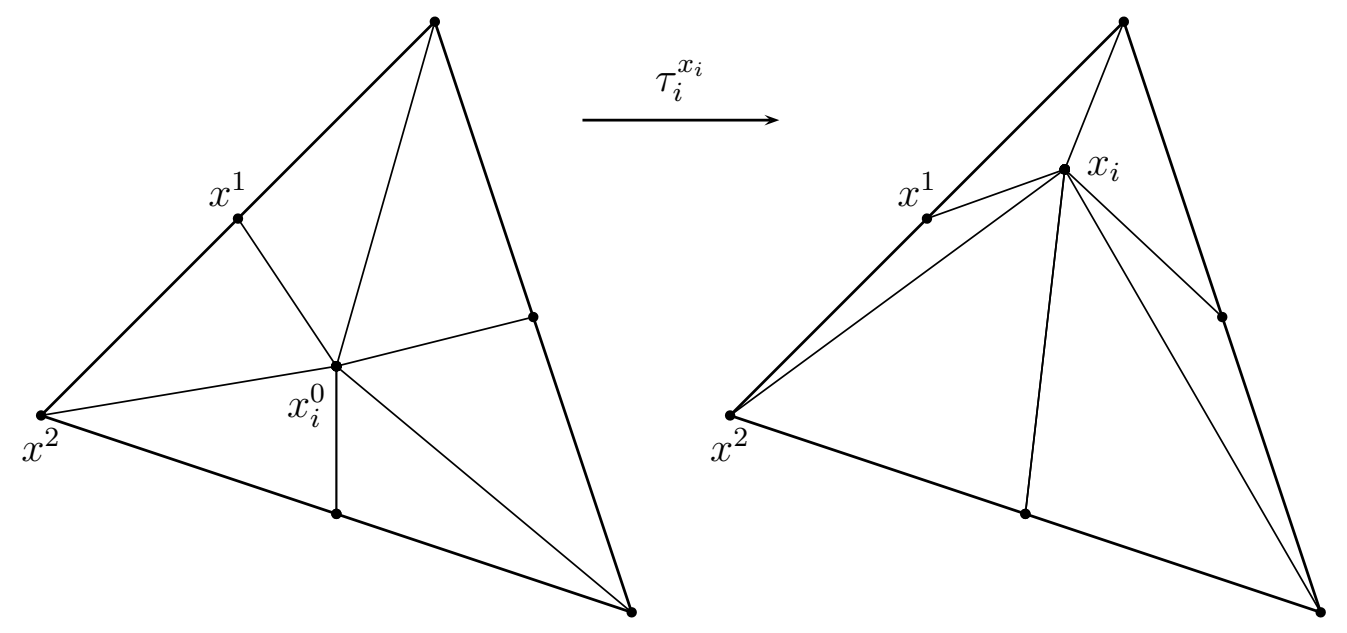

Figure 4: The homeomorphism $\tau_{i}^{x_{i}}=\tau\left(x_{i}, \cdot\right)$ when $X_{i}^{\prime}$ is a 2-simplex. 